\title{
The Long-Term Natural History of Trachomatous Trichiasis in The Gambia
}

\author{
Matthew J. Burton, ${ }^{1,2}$ Richard J. C. Bowman, ${ }^{1,3,4}$ Hannah Faal, $^{3}$ Esther A. N. Aryee, ${ }^{2}$ \\ Usman N. Ikumapayi, ${ }^{2}$ Neal D. E. Alexander, ${ }^{1}$ Richard A. Adegbola, ${ }^{2}$ David C. W. Mabey, ${ }^{1}$ \\ Allen Foster, ${ }^{1}$ Gordon J. Jobnson, ${ }^{1,5}$ and Robin L. Bailey ${ }^{1,2}$
}

Purpose. Trachoma is the leading infectious cause of blindness. However, there are few data on the natural history of trachomatous trichiasis to guide program planning or that investigate its pathogenesis.

Methods. A cohort of Gambians with trichiasis in one or both eyes who had declined surgery was observed. Clinical examinations were performed at baseline and 4 years later. Conjunctival swab samples were collected for Chlamydia trachomatis PCR and bacteriology.

RESults. One hundred fifty-four people were examined at baseline and 4 years later ( 241 nonsurgical eyes). At baseline 124 (52\%) eyes had major trichiasis (5+ lashes), 75 (31\%) minor trichiasis (1- 4 lashes), and 42 (17\%) no trichiasis. By 4 years, trichiasis had developed in 12 (29\%) of 42 previously unaffected eyes. Minor trichiasis progressed to major in $28(37 \%)$ of 75 eyes. New corneal opacification more commonly developed in eyes that had major (10\%) compared to minor (5\%) trichiasis at baseline. Bacterial infection was common (23\%), becoming more frequent with increasing trichiasis. C. trachomatis infection was rare (1\%). Conjunctival inflammation was common (29\%) and was associated with progressive trichiasis and corneal opacification.

Conclusions. Trichiasis progressed in the long-term in this environment, despite a low prevalence of $C$. trachomatis. Blinding corneal opacification develops infrequently, unless major trichiasis is present. Epilation and early surgery need to be formally compared for the management of minor trichiasis. The pathologic correlates and promoters of conjunctival inflammation need to be investigated. (Invest Ophthalmol Vis Sci. 2006;47:847-852) DOI:10.1167/iovs.05-0714

$\mathrm{T}$ rachoma is the leading infectious cause of blindness worldwide. It is estimated that approximately 2 million people are blind from the disease. ${ }^{1}$ It begins in childhood with recur-

From the ${ }^{1}$ International Centre for Eye Health, London School of Hygiene and Tropical Medicine, London, United Kingdom; ${ }^{2}$ Medical Research Council Laboratories, Fajara, The Gambia; the ${ }^{3}$ National Eye Care Programme, The Gambia.; ${ }^{4}$ CCBRT Disability Hospital, Dar Es Salaam, Tanzania; and the ${ }^{5}$ Division of Epidemiology and International Eye Health, Institute of Ophthalmology, London, United Kingdom.

Supported by a grant from The Wellcome Trust and the Burroughs Wellcome Fund.

Submitted for publication June 7, 2005; revised September 10, 2005; accepted January 11, 2006.

Disclosure: M.J. Burton, None; R.J.C. Bowman, None; H. Faal, None; E.A.N. Aryee, U.N. Ikumapayi, None; N.D.E. Alexander, None; R.A. Adegbola, None; D.C.W. Mabey, None; A. Foster, None; G.J. Johnson, None; R.L. Bailey, None

The publication costs of this article were defrayed in part by page charge payment. This article must therefore be marked "advertisement" in accordance with 18 U.S.C. $\$ 1734$ solely to indicate this fact.

Corresponding author: Matthew Burton, International Centre for Eye Health, London School of Hygiene and Tropical Medicine, Keppel Street, London WC1E 7HT, UK; matthew.burton@1shtm.ac.uk. rent episodes of chronic follicular conjunctivitis due to Chlamydia trachomatis infection. Chronic inflammation promotes tarsal conjunctival scarring, leading to entropion, trichiasis and corneal opacification. ${ }^{2-4}$ The World Health Organization (WHO) is leading a global effort to control blinding trachoma by 2020 through implementation of the SAFE strategy: Surgery for trichiasis, Antibiotic distribution for chlamydial infection, Facial cleanliness, and Environmental improvements to reduce transmission. ${ }^{5}$

There are few short-term and no long-term data on the natural history of trichiasis, its progression in different environments and incidence of corneal opacification at different stages of disease. ${ }^{6}$ Knowledge of what drives the cicatricial process especially in regions where $C$. trachomatis infection is less common is limited. The optimal management of early trichiasis remains unclear: Is it better to perform early surgery or to defer until a more severe problem develops? Trichiasis is probably the principal insult in the pathogenesis of trachomatous corneal opacification either directly through trauma or indirectly through secondary bacterial infection of the damaged cornea, although prospective data are limited. Other factors may contribute to corneal damage such as ocular dryness, and conjunctival inflammation. ${ }^{6,7} \mathrm{~A}$ better understanding of the natural history of trachomatous trichiasis could aid design of trachoma control programs, guide the timing of surgery, and identify potentially modifiable risk factors for disease progression. This study reports the four-year natural history of a group of Gambians with trachomatous trichiasis who had previously declined surgery. ${ }^{6,8,9}$ Risk factors for progressive disease are investigated.

\section{METHODS}

\section{Ethical Permission}

This study was approved by the Gambian Government/Medical Research Council Joint Ethics Committee and was conducted in accordance with the tenets of the Declaration of Helsinki. The nature of the study was explained and consent given before clinical assessment.

\section{Clinical Assessment}

Individuals identified with nonsurgically treated trichiasis during the course of earlier studies were revisited 4 years later. ${ }^{6,8}$ On both occasions patients were assessed by ophthalmologists experienced in the examination of patients with trachoma using $2.5 \times$ binocular loupes and a torch (baseline: RJCB. 4 years: MJB). Baseline clinical data were available for each patient including Snellen visual acuity, trichiasis severity (major: $5+$ lashes; minor: $1-4$ lashes) and the presence of visually significant corneal opacification. At follow-up patients were asked whether they epilated and if so how frequently. The visual acuity was remeasured using reduced logMAR (logarithm of the minimum angle of resolution). ${ }^{10}$ Clinical signs were graded using the WHO trachoma grading system. ${ }^{11}$ Lashes touching the cornea and other parts of the eye in primary position were counted separately. Evidence of epilation was noted: broken/regrowing lashes or sections of eyelid denuded of lashes. If evidence of epilation was found the extent of the 
Table 1. Trichiasis Status at Baseline and Follow-up in Each of the 241 Eyes

\begin{tabular}{lccc}
\hline & \multicolumn{3}{c}{ Trichiasis Status at Follow-up } \\
\cline { 2 - 4 } & $\begin{array}{c}\text { No } \\
\text { Trichiasis }\end{array}$ & $\begin{array}{c}\text { Minor } \\
\text { Trichiasis }\end{array}$ & $\begin{array}{c}\text { Major } \\
\text { Trichiasis }\end{array}$ \\
\hline Trichiasis status at baseline & & & \\
No trichiasis & 30 & 6 & 6 \\
Minor trichiasis & 16 & 31 & 28 \\
Major trichiasis & 1 & 10 & 113 \\
\hline
\end{tabular}

epilation was recorded as less than or more than half the length of the eyelid. Corneal opacification was considered visually significant if part of the pupil margin was obscured (CC2/CC $3^{11}$ or $\left.\mathrm{CO}^{12}\right)$. Conjunctival inflammation was considered significant if there were prominent papillae and haziness of the tarsal blood vessel (P2 or P3). ${ }^{11}$

At the follow-up assessment conjunctival swab samples were collected. The conjunctiva was anesthetized with proxymetacaine $0.5 \%$ eye drops (Minims; Chauvin Pharmaceuticals, Romford, UK). An inferior fornix swab sample was collected and immediately placed into a sterile tube containing STGG broth (skimmed milk-tryptone-glycerolglucose broth) for bacterial culture. A second swab sample for $C$. trachomatis PCR was collected from the upper tarsal conjunctiva and placed in a dry tube. Both sets of samples were kept on ice before transfer to $-70^{\circ} \mathrm{C}$ or $-20^{\circ} \mathrm{C}$ freezers, respectively, later the same day. Individuals with trichiasis were offered surgery by the Gambian National Eye Care Programme.

\section{Polymerase Chain Reaction for C. trachomatis}

Conjunctival samples were tested for $C$. trachomatis with a commercial qualitative PCR-based assay (Amplicor CT/NG Test; Roche Molecular Systems, Branchburg, NJ), with previously described modifications. ${ }^{13}$

\section{General Bacteriology}

Conjunctival samples were plated on: Blood Agar (aerobic and anaerobic), McConkey Agar (aerobic), Gentamicin Blood Agar and Bacitracin Chocolate Agar. The Gentamicin Blood Agar and the Bacitracin Chocolate Agar were placed in a 5\% carbon dioxide incubator. All plates were incubated at $37^{\circ} \mathrm{C}$ for 48 hours and isolated organisms were identified using standard bacteriologic techniques. Staphylococcus epidermidis and Bacillus were excluded from the analysis, as they were considered commensal rather than pathogenic at this site.

\section{Data Analysis}

Data were analyzed on computer (STATA ver. 7; STATA Corp., College Station, TX). Change in visual acuity before and after surgery was assessed using paired two-sided $t$-tests. The baseline Snellen visual acuity was converted to a logMAR score. ${ }^{10}$ For eyes whose visual acuity was counting fingers or less, logMARs were attributed as follows: counting fingers 2.0, hand movements 2.5, and perception of light 3.0. For the purposes of analysis eyes were considered to have major

TABLE 2. Severity of Trichiasis at Follow-up

\begin{tabular}{lcc}
\hline Number of Lashes Touching Eye & $\begin{array}{c}\text { Subjects } \\
(\boldsymbol{n})\end{array}$ & $\mathbf{\%}$ \\
\hline 0 (no evidence of epilation) & 47 & 19.5 \\
0 (evidence of epilation) & 26 & 10.8 \\
$1-4$ & 66 & 27.4 \\
$5-9$ & 54 & 22.4 \\
$10-19$ & 31 & 12.8 \\
$20^{+}$ & 17 & 7.1 \\
Total & 241 & \\
\hline
\end{tabular}

TABLE 3. Corneal Opacification Status at Baseline and Follow-up

\begin{tabular}{lrrr}
\hline & \multicolumn{2}{c}{ Follow-up CO } & \\
\cline { 2 - 3 } & + & - & Total \\
\hline Baseline CO & & \\
+ & 18 & 12 & 30 \\
- & 16 & 195 & 211 \\
Total & 34 & 207 & 241 \\
\hline \multicolumn{2}{l}{,+ present; - , absent. } & &
\end{tabular}

trichiasis if they had five or more lashes in contact with the globe or there was extensive epilation involving more than half the length of the eyelid. Eyes were considered to have minor trichiasis if there were 1 to 4 lashes touching the globe or there was evidence of epilation which involved less than half the length of the eyelid, without lashes touching the globe. Univariate odds ratios were calculated for the association between trichiasis and risk factors. Multivariable logistic regression models were developed for trichiasis and its progression. Generalized estimating equations (GEE) were used to adjust for the correlation between eyes in bilateral cases for both univariate and multivariate analyses.

\section{Results}

\section{Patient Characteristics}

At baseline a total of 220 individuals with trachomatous trichiasis in at least one eye were identified and offered surgery. ${ }^{6,8}$ Four years later we attempted to recontact them. In all 153 (70\%) of 220 individuals were located and reexamined. Of the 67 who were not reassessed 41 had died, 24 were untraceable, and 2 refused. Trichiasis surgery had been performed on one eye in 65 of 153 subjects, leaving 241 nonsurgically treated eyes eligible for inclusion in this study. The following analysis is based on these 241 eyes only. The majority (109/153; 71\%) were women; they had a mean age of $59 \pm 13.6$ years (SD) and were predominantly Mandinka ethnicity (124; 81\%).

\section{Trichiasis Status}

At baseline 124 (51.5\%) of 241 eyes had major trichiasis, 75 (31.1\%) had minor trichiasis, and 42 (17.4\%) had no trichiasis. At follow-up, 147 (61\%) 241 eyes had major trichiasis, 47 (19.5\%) had minor trichiasis, and 47 (19.5\%) had no trichiasis. Baseline and follow-up trichiasis status were compared (Table 1): no change in $174(72.2 \%)$ eyes, deterioration in 40 (16.6\%), and improvement in 27 (11.2\%). Minor trichiasis progressed to major trichiasis in $28(37.3 \%)$ of 75 eyes; whereas trichiasis developed in 12 (28.6\%) of 42 previously unaffected eyes. Trichiasis was not found in 17 eyes at follow-up that were affected at baseline (16/17 minor trichiasis at baseline). The distribution of trichiasis severity at follow-up is shown in Table 2. Subjects self-reported regularly epilating 165 (68.5\%) of 241 eyes. Epilation was more frequently self-reported for eyes found to have increasing severity of trichiasis on examination (OR 3.48; 95\% CI, 2.39-5.06; $P<0.001$ ). Among those with major trichiasis at baseline the commonest reasons for not having surgery were fear (37\%), lack of time (12\%), and cost $(10 \%)$. At the time of follow-up 36 individuals with major trichiasis accepted surgery.

\section{Corneal Opacification}

At baseline 30 (12.4\%) of 241 eyes had visually significant corneal opacification (CO). At follow-up, a similar proportion of eyes had CO $(34 / 241 ; 14.1 \%)$. However, there was some change in CO status between baseline and follow-up (Table 3), 
Table 4. Corneal Opacification by Trichiasis Status at Baseline and Follow-up

\begin{tabular}{lcc}
\hline & $\begin{array}{c}\text { Corneal Opacification } \\
\text { Present }(\boldsymbol{n}=\mathbf{2 4 1})\end{array}$ & $\%$ \\
\hline Baseline* & & \\
$\quad$ No trichiasis & $0 / 42$ & 0 \\
Minor trichiasis & $5 / 75$ & 6.7 \\
$\quad$ Major trichiasis & $25 / 124$ & 20.2 \\
Follow-upt & $0 / 47$ & 0 \\
$\quad$ No trichiasis & $3 / 47$ & 6.4 \\
Minor trichiasis & $31 / 147$ & 21.1 \\
Major trichiasis & & \\
\hline
\end{tabular}

$*$ Association between increasing trichiasis and CO: odds ratio: 4.51. 95\% CI: $1.89-11.2$.

†Association between increasing trichiasis and CO: odds ratio: 5.57. $95 \%$ CI: $1.92-16.2$.

with 16 (6.6\%) eyes developing CO and 12 eyes improving CO. At both time points, $\mathrm{CO}$ was only found in eyes with trichiasis and was more common with major trichiasis (Table 4). New cases of $\mathrm{CO}$ were associated with increasing trichiasis severity at follow-up (14/16 had major trichiasis, 2/16 minor trichiasis. OR 3.96; 95\% CI, 1.44-10.9; $P=0.008$ ) and conjunctival inflammation (OR 3.17; 95\% CI, 1.23-8.13; $P=0.017$ ). Bacterial infection was not associated with $\mathrm{CO}$ at follow-up. Of the 117 eyes with no or only minor trichiasis at baseline, six (5.1\%) developed new CO by follow-up. Of these six eyes, four had developed major and two had minor trichiasis by follow-up. Of the 99 eyes with major trichiasis but no $\mathrm{CO}$ at baseline, 10 developed CO by follow-up. Of the 12 eyes in which the CO resolved 9 had major trichiasis on both occasions, 1 had minor trichiasis, and 2 had resolution of trichiasis.

\section{Visual Acuity}

Paired baseline and follow-up visual acuity was available for 214 eyes (Table 5). Overall mean reduction in visual acuity was 0.222 logMAR units $(P<0.0001$, paired, two-sided $t$-test $)$. The reduction in vision tended to be less (not statistically significant) in eyes without trichiasis at follow-up (0.147 logMAR) compared with eyes with major trichiasis (0.299 logMAR). The reduction in vision was significantly greater in eyes that developed new CO (0.648 logMAR; $P=0.007)$. At baseline 20 (9.3\%) of 214 of eyes had a visual acuity of less than $3 / 60$ (Table 5). By follow-up, an additional 29 eyes had deteriorated to less than $3 / 60$. At follow-up CO was associated with visual acuities of less than 3/60 (OR 6.04; 95\% CI, 2.64-13.8; $P<0.001$ ). However, only 6 of 29 eyes that deteriorated to $<3 / 60$ had CO at follow-up, suggesting that most of the reduction in vision was due to other causes, such as cataract.

\section{Conjunctival Infection}

C. trachomatis PCR testing was performed for 146 of 153 subjects. Two (1.4\%) had positive results. Bacterial culture

TABLE 5. Visual Acuity at Baseline and Follow-up

\begin{tabular}{llrlllr}
\hline & & \multicolumn{2}{c}{ Baseline } & & \multicolumn{2}{c}{ Follow-up } \\
\cline { 3 - 4 } \cline { 7 - 8 } $\begin{array}{l}\text { Visual } \\
\text { Acuity }\end{array}$ & & Number & $\%$ & & Number & $\%$ \\
\hline $0.0-0.48$ & $(6 / 6-6 / 18)$ & 105 & 49.1 & & 60 & 28.1 \\
$<0.48-1.0$ & $(<6 / 18-6 / 60)$ & 73 & 34.1 & & 88 & 41.1 \\
$<1.0-1.3$ & $(<6 / 60-3 / 60)$ & 16 & 7.5 & & 21 & 9.8 \\
$<1.3$ & $(<3 / 60)$ & 20 & 9.3 & & 45 & 21.0 \\
\hline
\end{tabular}

$$
n=214 \text { eyes }
$$

TABLE 6. Bacterial Isolation Rate by Trichiasis Status at Follow-up, from 147 Samples

\begin{tabular}{lcc}
\hline Trichiasis Status & Bacterial Isolates & $\%$ \\
\hline No trichiasis & $0 / 7$ & 0 \\
Minor trichiasis & $3 / 30$ & 10.0 \\
Major trichiasis & $31 / 110$ & 28.2 \\
\hline
\end{tabular}

Association between increasing trichiasis and bacterial isolation: odds ratio: 4.05 . 95\% CI: $1.25-13.1$.

samples were collected from 147 of 241 eyes. Pathologic isolates were grown from 34 of 147 (23\%) samples. Isolation rates increased with increasing severity of trichiasis (Table 6). Several different organisms were cultured (Table 7).

\section{Risk Factors for Trichiasis and Its Progression}

Univariate associations between major trichiasis and various risk factors at follow-up for 241 eyes are shown in Table 8. Conjunctival inflammation (grades P2 or P3), bacterial infection and age were associated with trichiasis. A subgroup analysis of risk factors for trichiasis progression was performed, comparing individuals with stable disease $(n=77)$ with those who developed new or more trichiasis $(n=40)$, excluding individuals who had major trichiasis at baseline (Table 8). In this subgroup analysis, conjunctival inflammation was associated with progressive trichiasis.

Multivariable logistic regression models for associations between risk factors and major trichiasis and progressive trichiasis were developed (Table 9). These identified significant associations between major trichiasis and increasing age, conjunctival inflammation and bacterial infection. No significant associations were identified for progression in trichiasis.

\section{Baseline Data on Those Who Died before Follow-up}

The mean age of those who died between baseline and follow-up was $62 \pm 12.2$ years of whom $62 \%$ were women. Major trichiasis had been observed in $64 \%$ of eyes at baseline and CO was found in $25 \%$.

\section{Discussion}

\section{Clinical Natural History}

This study reports the 4-year natural history of a cohort of Gambians with trachomatous trichiasis. During this period some individuals had progressive disease with $29 \%$ of unilateral cases becoming bilateral and $37 \%$ of eyes with minor trichiasis developing major trichiasis. An earlier study from The Gambia found faster rates of progression: during a 1-year period $46 \%$ of unilateral cases became bilateral and 33\% of minor trichiasis progressed to major trichiasis. ${ }^{6}$ The apparent slowing of dis-

TABLE 7. Pathological Conjunctival Bacterial Isolates, from 147 Samples

\begin{tabular}{lcr}
\hline \multicolumn{1}{c}{ Organism } & Number & $\%$ \\
\hline Streptococcus pneumoniae & 16 & 47 \\
Staphylococcus aureus & 7 & 20 \\
Streptococcus spp & 5 & 15 \\
Streptococcus group C & 2 & 6 \\
Pseudomonas aeruginosa & 1 & 3 \\
Moraxella & 1 & 3 \\
Klebsiella & 1 & 3 \\
Streptococcus viridians & 1 &
\end{tabular}


TABLE 8. Univariate Associations

\begin{tabular}{|c|c|c|c|c|c|c|}
\hline \multirow[b]{2}{*}{ Variable (at Follow-up) } & \multicolumn{3}{|c|}{ 5+ Lashes } & \multicolumn{3}{|c|}{ Progressive TT } \\
\hline & OR & $95 \% \mathrm{CI}$ & $\boldsymbol{P}$ & OR & $95 \% \mathrm{CI}$ & $\boldsymbol{P}$ \\
\hline \multicolumn{7}{|l|}{ Age $(y)$} \\
\hline$<50$ & 1 & - & - & 1 & - & - \\
\hline $50-59$ & 2.05 & $0.87-4.87$ & 0.102 & 1.76 & $0.59-5.29$ & 0.312 \\
\hline $60-69$ & 1.91 & $0.81-4.51$ & 0.138 & 0.85 & $0.24-2.96$ & 0.798 \\
\hline $70+$ & 2.39 & $1.07-5.33$ & 0.033 & 0.74 & $0.24-2.28$ & 0.595 \\
\hline Sex (female) & 0.68 & $0.35-1.33$ & 0.263 & 0.74 & $0.29-1.87$ & 0.522 \\
\hline Ethnicity (non-Mandinka) & 0.52 & $0.24-1.10$ & 0.088 & 0.78 & $0.28-2.18$ & 0.641 \\
\hline C. trachomatis infection & 2.23 & $0.94-5.26$ & 0.068 & 2.61 & $0.74-9.17$ & 0.136 \\
\hline Bacterial infection & 3.74 & $1.15-12.0$ & 0.027 & 2.43 & $0.40-14.9$ & 0.337 \\
\hline Conjunctival inflammation & 3.53 & $1.81-6.85$ & 0.001 & 3.07 & $1.23-7.70$ & 0.017 \\
\hline
\end{tabular}

Results of analyses are shown for various risk factors at follow-up and major trichiasis, and for those with progressive trichiasis, adjusting for the correlation between eyes in bilateral cases by generalized estimating equations.

ease progression in The Gambia may be attributable to the reduction in prevalence of active trachoma and C. trachomatis infection in recent years. ${ }^{14}$ However, our study demonstrates that despite lower levels of active trachoma the cicatricial complications continue to progress. This observation is of programmatic significance as ongoing surveillance and surgical treatment for trichiasis will be needed in communities where active trachoma was formerly endemic.

We found that $10 \%$ of eyes with major trichiasis at baseline developed new CO by follow-up, whereas, CO developed in $5 \%$ of eyes with minor or no trichiasis at baseline. However, most of these eyes with new $\mathrm{CO}$ had also progressed to major trichiasis by 4 years. This visual loss might have been prevented with timely surgery. As individuals with major trichiasis are a high-risk group for developing corneal damage and visual loss, efforts to promote surgery should be concentrated on them. Where surgery is declined, follow-up is needed.

Several national trachoma control programs, including The Gambia, advise patients to epilate minor trichiasis and postpone surgery until major trichiasis develops. ${ }^{9}$ They reason that only a small minority of individuals with minor trichiasis develop $\mathrm{CO}$, especially when the inturned lashes are peripheral. Other programs take the view that any degree of trichiasis is an indication for surgery, especially if the patient has limited access to surgery. They argue that the disease may progress and blinding corneal opacification could develop before the patient is reviewed again. There are no published studies comparing the outcome of vision and corneal opacification for these alternative strategies. A randomized controlled trial of electrolysis, cryoablation and tarsal rotation surgery for minor trichiasis found no difference in visual acuity between these alternatives, although the surgical group had a much lower postoperative trichiasis rate. ${ }^{15}$ Individuals with minor trichiasis often decline surgery. ${ }^{6,9}$ Therefore, it would be useful to know whether by improving the quality of epilation through training and equipping community volunteers with good eyesight, the long-term visual outcome is as good as early surgery.

Two different observers performed baseline and follow-up examinations, and it was not possible to assess potential interobserver variability. Both ophthalmologists have extensive experience in the grading of trachoma. The principal clinical measures compared between the two time points in this study, the presence of trichiasis and corneal opacification, have clear, objective definitions. Studies have demonstrated very good intra- and interobserver reliability. ${ }^{16}$ Therefore, we believe the potential for misclassification of these particular signs is limited. However, disease manifestations may vary with time.

In common with all studies of trachoma the grading of trichiasis is complicated by the practice of epilation. The patients reported in this study frequently performed epilation. This could potentially lead to an underestimate of the prevalence and severity of trichiasis and probably explains why some individuals were observed to have less trichiasis at follow-up. Long-term repeated epilation may also result in madarosis. This "noise" in the clinical phenotype supports the use of fairly broad categories for the classification of trichiasis, such as major and minor trichiasis. In addition, we recorded evidence of epilation being practiced.

Several cases of CO observed at baseline had improved by follow-up. There are several potential explanations for this. It could be due to differences in interpretation of clinical observations between observers. The WHO defines CO as an "easily

TABLE 9. Multivariable Logistic Regression Models

\begin{tabular}{|c|c|c|c|c|c|c|}
\hline \multirow[b]{2}{*}{ Variable (at Follow-up) } & \multicolumn{3}{|c|}{ 5+ Lashes } & \multicolumn{3}{|c|}{ Progressive TT } \\
\hline & OR & $95 \% \mathrm{CI}$ & $\boldsymbol{P}$ & OR & $95 \%$ CI & $\boldsymbol{P}$ \\
\hline \multicolumn{7}{|l|}{ Age (years) } \\
\hline$<50$ & 1 & - & - & 1 & - & - \\
\hline $50-59$ & 2.54 & $0.83-7.72$ & 0.101 & 3.00 & $0.67-13.5$ & 0.152 \\
\hline $60-69$ & 5.31 & $1.35-20.9$ & 0.017 & 2.55 & $0.37-17.7$ & 0.345 \\
\hline $70+$ & 3.26 & $1.07-9.97$ & 0.038 & 1.23 & $0.23-6.61$ & 0.808 \\
\hline Sex (female) & 1.38 & $0.55-3.48$ & 0.489 & 1.37 & $0.35-5.30$ & 0.652 \\
\hline Conjunctival inflammation & 4.18 & $1.20-14.6$ & 0.025 & 2.04 & $0.54-7.64$ & 0.288 \\
\hline Bacterial infection & 2.72 & $1.05-7.05$ & 0.040 & 4.33 & $0.62-30.1$ & 0.138 \\
\hline
\end{tabular}

Results are shown for associations between major trichiasis and various factors, adjusting for the correlation between eyes in bilateral cases by generalized estimating equations. 
visible corneal opacity over the pupil." However, the size of the pupil will vary in different levels of ambient light. This could lead to variable classification of opacities that are not absolutely central. Alternatively, some opacities may have faded with time, if the causative factor such as secondary bacterial infection is reduced or resolves.

During the 4 years between baseline and follow-up, 19\% of those originally assessed died. These individuals tended to be slightly older and have slightly more advanced disease at baseline (major trichiasis and corneal opacification), potentially leading to an underestimate in disease progression.

\section{Pathogenesis of Blinding Trachoma}

We found that major trichiasis at follow-up was associated with increasing age, conjunctival inflammation and bacterial infection. Progression of trichiasis in the subgroup without major trichiasis at baseline was associated with conjunctival inflammation at follow-up. Several factors may promote the development of trachomatous corneal opacification: trichiasis, bacterial infection, ocular surface dryness and conjunctival inflammation. At both time points in this study CO was only found in eyes with trichiasis and new $\mathrm{CO}$ was associated with increasing severity of trichiasis, suggesting that this is the principal insult. In addition, we identified an association between new corneal opacities and conjunctival inflammation. Other studies from the Gambia have found associations between CO and severe trichiasis, bacterial infection and conjunctival inflammation. ${ }^{4,6,7}$

Chronic inflammation of the conjunctiva is probably the central event in the pathogenesis of the cicatricial complications of trachoma. Persistent severe inflammatory trachoma (TI) in children, triggered by recurrent chlamydial infection, has been associated with the development of conjunctival scarring and trichiasis later in life. ${ }^{2,3}$ Adults with conjunctival scarring and trichiasis frequently have conjunctival inflammation. ${ }^{7,17}$ The presence of conjunctival scarring may limit the area of the tarsal conjunctival surface that can develop an inflammatory phenotype. Therefore, for the purposes of analysis we considered the conjunctiva to be inflamed if the diagnostic criteria of grades P2 or P3 of the World Health Organization (WHO) system are met. ${ }^{18}$ It is uncertain what drives ongoing inflammation in late stage disease. Several factors may contribute: chlamydial or bacterial infections, mechanical irritation from inturned lashes, a dry ocular surface or a chronic immunologic process. In the present study conjunctival inflammation was associated with major trichiasis but not $C$. trachomatis or bacterial infection. We have previously found conjunctival inflammation to be independently associated with both more severe trichiasis $(10+$ lashes $)$ and bacterial infection but not chlamydial infection. ${ }^{7} C$. trachomatis may be more important in meso- and hyperendemic regions. In a study of recurrent trichiasis after surgery in Tanzania conjunctival inflammation was weakly associated with $C$. trachomatis infection; $10 \%$ of those with conjunctival inflammation had chlamydial infection. ${ }^{18}$

Whatever the cause, it is probable that conjunctival inflammation observed in these studies of people with late stage trachoma reflects an ongoing inflammatory cicatricial process. In younger individuals with clinically active trachoma, conjunctival inflammation is associated with marked inflammatory cell infiltrates (macrophages, lymphocytes, and neutrophils) and increased expression of proinflammatory cytokines such as interleukin- $1 \beta$ and tumor necrosis factor- $\alpha .{ }^{19,20}$ These probably activate proteolytic enzymes, the matrix metalloproteinases (MMP), which can degrade the extracellular matrix and facilitate tissue remodelling and scar formation. In particular, MMP-9, which has been implicated in a number of cicatricial diseases, is expressed at increased levels in inflamed conjunctiva. ${ }^{20}$ Some of these conjunctiva-derived factors may be released into the tear film or directly onto the corneal surface promoting scar formation. There is evidence which indicates that matrix metalloproteinases, particularly MMP-9, may be important in corneal damage in various inflammatory and infectious diseases and may therefore be important in trachoma. $^{21-23}$

Bacterial infection is common in eyes with trichiasis and is significantly reduced after trichiasis surgery. ${ }^{7,17}$ It is likely that trichiasis facilitates recurrent inoculation of the conjunctival sac with bacteria and inhibits the resolution of infection. Other factors may also compromise the protective mechanisms of the trachomatous ocular surface, such as reduced tear production and increased keratinization. As discussed above, bacterial infection is frequently associated with conjunctival inflammation in eyes with trichiasis, raising the possibility that it may contribute to progressive inflammatory cicatricial changes in late stage disease. ${ }^{7}$ Bacterial infection may promote corneal opacification either through secondary infection of damaged cornea or possibly indirectly through promotion of conjunctival inflammation. ${ }^{7}$ In earlier studies, but not in the present one, we found an association between $\mathrm{CO}$ and bacterial infection. ${ }^{6,7}$ To further evaluate the role of bacterial infection in blinding trachoma a prospective study of individuals with trichiasis refusing surgery is needed, which intensively assesses the effect of bacterial infection on disease progression. If bacterial infection does prove to be significant then it may be useful to develop targeted interventions.

In conclusion, we found that many individuals with trichiasis had disease progression. This process continues despite reductions in the prevalence of active trachoma, indicating the need for ongoing surveillance. CO developed only in the presence of trichiasis. Individuals with major trichiasis are at the highest risk of disease progression. Therefore control program efforts should focus initially on promoting surgery in this group. Those refusing surgery should be followed-up and recounseled. Conjunctival inflammation was associated with progressive trichiasis and $\mathrm{CO}$, possibly reflecting an active cicatricial process. The stimulants for inflammation in late-stage disease remain uncertain.

\section{Acknowledgments}

The authors thank the ophthalmic nurses of the Gambian National Eye Care Programe and the field staff from the Medical Research Council Laboratories for their hard work, often under quite challenging conditions.

\section{References}

1. Resnikoff S, Pascolini D, Etya'ale D et al. Global data on visual impairment in the year 2002. Bull World Health Organ. 2004;82: $844-851$

2. Dawson CR, Marx R, Daghfous T, Juster R, Schachter J. What clinical signs are critical in evaluating the intervention in trachoma? 1990;271-278.

3. West SK, Munoz B, Mkocha H, Hsieh YH, Lynch MC. Progression of active trachoma to scarring in a cohort of Tanzanian children. Opbthalmic Epidemiol. 2001;8:137-144.

4. Bowman RJ, Jatta B, Cham B et al. Natural history of trachomatous scarring in The Gambia: results of a 12-year longitudinal follow-up. Opbthalmology. 2001;108:2219-2224.

5. World Health Organisation. Global elimination of blinding trachoma. Resolution WHA 51.11 adopted by the World Health Assembly 16 May 1998.

6. Bowman RJ, Faal H, Myatt M et al. Longitudinal study of trachomatous trichiasis in the Gambia. Br J Opbthalmol. 2002;86:339343. 
7. Burton MJ, Kinteh F, Jallow O et al. A randomised controlled trial of azithromycin following trichiasis surgery in The Gambia. $\mathrm{BrJ}$ Ophthalmol. 2005 (In Press)

8. Bowman RJ, Soma OS, Alexander N et al. Should trichiasis surgery be offered in the village? A community randomised trial of village vs. health centre-based surgery. Trop Med Int Health. 2000;5:528 533.

9. Bowman RJ, Faal H, Jatta B et al. Longitudinal study of trachomatous trichiasis in The Gambia: barriers to acceptance of surgery. Invest Ophthalmol Vis Sci. 2002;43:936-940.

10. Rosser DA, Laidlaw DA, Murdoch IE. The development of a "reduced logMAR" visual acuity chart for use in routine clinical practice. Br J Opbthalmol. 2001;85:432-436.

11. Dawson CR, Jones BR, Tarizzo ML. Guide to Trachoma Control. Geneva. World Health Organization. 1981.

12. Thylefors B, Dawson CR, Jones BR, West SK, Taylor HR. A simple system for the assessment of trachoma and its complications. Bull World Health Organ. 1987;65:477-483.

13. Burton MJ, Holland MJ, Faal N et al. Which Members of a Community Need Antibiotics to Control Trachoma? Conjunctival Chlamydia trachomatis Infection Load in Gambian Villages. Invest Opbthalmol Vis Sci. 2003;44:4215-4222.

14. Dolin PJ, Faal H, Johnson GJ, Ajewole J, Mohamed AA, Lee PS. Trachoma in The Gambia. Br J Opbthalmol. 1998;82:930-933.

15. Reacher MH, Munoz B, Alghassany A, Daar AS, Elbualy M, Taylor HR. A controlled trial of surgery for trachomatous trichiasis of the upper lid. Arch Ophthalmol. 1992;110:667-674.
16. Taylor HR, West SK, Katala S, Foster A. Trachoma: evaluation of a new grading scheme in the United Republic of Tanzania. Bull World Health Organ. 1987;65:485-488.

17. Burton MJ, Bowman RJ, Faal $\mathrm{H}$ et al. Long term outcome of trichiasis surgery in the Gambia. Br J Opbthalmol. 2005;89:575579.

18. West ES, Mkocha $\mathrm{H}$, Munoz B et al. Risk factors for postsurgical trichiasis recurrence in a trachoma-endemic area. Invest Opbthalmol Vis Sci. 2005;46:447-453.

19. El Asrar AM, Van den Oord JJ, Geboes K, Missotten L, Emarah MH, Desmet V. Immunopathology of trachomatous conjunctivitis. Br J Opbthalmol. 1989;73:276-282.

20. Burton MJ, Bailey RL, Jeffries D, Mabey DC, Holland MJ. Cytokine and fibrogenic gene expression in the conjunctivas of subjects from a gambian community where trachoma is endemic. Infect Immun. 2004;72:7352-7356.

21. Leonardi A, Brun P, Abatangelo G, Plebani M, Secchi AG. Tear levels and activity of matrix metalloproteinase (MMP)-1 and MMP-9 in vernal keratoconjunctivitis. Invest Ophthalmol Vis Sci. 2003; 44:3052-3058.

22. Smith VA, Rishmawi H, Hussein H, Easty DL. Tear film MMP accumulation and corneal disease. BrJ Ophthalmol. 2001;85:147153.

23. Xue ML, Wakefield D, Willcox MD et al. Regulation of MMPs and TIMPs by IL-1beta during corneal ulceration and infection. Invest Ophthalmol Vis Sci. 2003;44:2020-2025. 